\title{
YAP promotes the proliferation and migration of colorectal cancer cells through the Glut3/AMPK signaling pathway
}

\author{
LINHUA JIANG*, JIAWEN ZHANG*, QIXUAN XU*, BIN WANG, YIZHOU YAO, LIANG SUN, \\ XUCHAO WANG, DIYUAN ZHOU, LING GAO, SHIDUO SONG and XINGUO ZHU
}

Department of General Surgery, The First Affiliated Hospital of Soochow University, Suzhou, Jiangsu 215006, P.R. China

Received October 29, 2020; Accepted February 2, 2021

DOI: $10.3892 /$ ol.2021.12573

\begin{abstract}
Yes-associated protein (YAP), as a major downstream effector in the Hippo signaling pathway, is considered as an oncogene in cancer. The present study aimed to investigate the potential role of YAP in the development and progression of colorectal cancer (CRC). The mRNA and protein expression levels of YAP in human CRC tissue samples and adjacent normal tissue were analyzed using public databases, as well as clinical samples. The potential roles of YAP and the underlying mechanism regulating the proliferation and migration of CRC cells were examined using genetic manipulation in vitro. The correlation between the expression of the YAP gene and epithelial-to-mesenchymal transition (EMT) markers was investigated in order to determine the mechanism underlying the observed effects of YAP. YAP mRNA expression levels were significantly upregulated in CRC tissue compared with in normal tissue, as determined using datasets obtained from Oncomine. Similarly, in clinical samples, the protein expression levels of YAP were significantly upregulated in CRC tissue samples compared with in normal tissue samples. YAP knockdown inhibited the proliferation and migration of CRC cells in vitro, whereas its overexpression resulted in the opposite effect. The expression levels of the YAP gene were positively correlated with those of EMT markers (such as vimentin and $\mathrm{N}$-cadherin) and EMT-inducing transcription factors (such as Snail1, Slug and zinc finger E-box binding homeobox 1 and 2) in CRC samples from Gene Expression Profiling Interactive Analysis. Furthermore, YAP silencing increased the protein expression of E-cadherin and decreased that of vimentin in CRC cells. By contrast, the overexpression of YAP had the opposite effect. YAP promoted the glucose
\end{abstract}

Correspondence to: Dr Xinguo Zhu, Department of General Surgery, The First Affiliated Hospital of Soochow University, 188 Shizi Street, Suzhou, Jiangsu 215006, P.R. China

E-mail: zhuxinguo1970@163.com

\section{${ }^{*}$ Contributed equally}

Key words: Yes-associated protein, proliferation, migration, epithelialto-mesenchymal-transition, colorectal cancer transporter 3 (Glut3)/AMP-activated protein kinase (AMPK) signaling pathway in CRC cells. In conclusion, YAP promoted the proliferation and migration of CRC cells, as well as the expression of EMT markers, possibly by regulating the Glut3/AMPK signaling pathway.

\section{Introduction}

Colorectal cancer (CRC) is one of the most common types of malignant cancer of the gastrointestinal tract and was ranked as the fourth leading cause of cancer-associated deaths worldwide in 2019 (1,2). Liver metastasis often develops in patients with CRC. Only $15-20 \%$ of patients with CRC with liver metastases are considered resectable, resulting in low survival rates (3). In China, CRC is the fourth most common type of cancer and fifth leading cause of cancer-related deaths, and a calculated 370,000 newly-diagnosed patients and 191,000 deaths occurred in 2015 (4). Moreover, the incidence and mortality rates have continuously increased over the last decades (1). Therefore, elucidating the molecular mechanisms underlying the progression and metastasis of $\mathrm{CRC}$ and identifying new biomarkers for the treatment of $\mathrm{CRC}$ is essential.

In mammals, the Hippo signaling pathway is involved in tissue repair, cell regeneration, proliferation and apoptosis. Impaired Hippo signaling contributes to the development and progression of cancer, including CRC (5-8). Yes-associated protein (YAP) is a major downstream effector in the Hippo signaling pathway (5). Through a kinase cascade, the Hippo signaling pathway results in the phosphorylation of YAP and inactivates it by cytoplasmic retention or proteasomal degradation (5). This prevents YAP translocation to the nucleus, where it normally functions as a transcriptional co-activator by interacting with TEA domain family members 1-4 and other transcription factors (5). The YAP gene has been proposed as a candidate oncogene, which may be closely associated with the initiation and progression of several types of cancer, including pancreatic cancer (9), breast cancer (10), hepatocellular carcinoma (11), esophageal cancer (12), cervical cancer (13), ovarian cancer $(14)$ and gastric cancer $(15,16)$. Recently, several studies have focused on the role of YAP in CRC. These studies demonstrated that YAP was overexpressed in CRC tissue, and promoted the proliferation, migration and epithelial-to-mesenchymal transition (EMT) of CRC cells (17-20). However, the 
underlying mechanism remains unclear and requires further study.

In the present study, the mRNA and protein expression levels of YAP in human CRC tissue and adjacent normal tissue were examined using public datasets and western blot analysis, and their potential role in the proliferation and migration of CRC cells was investigated in vitro. Moreover, the correlation of YAP with EMT-related genes was studied and confirmed using western blot analysis. In vitro studies further illustrated that YAP functioned as a regulator of glucose transporter 3 (Glut3)/AMP-activated protein kinase (AMPK) in order to mediate the proliferation and migration of CRC cells. Thus, YAP may serve as a candidate for the development of new strategies for CRC treatment.

\section{Materials and methods}

Clinical data and CRC tissue samples. Clinical data were obtained from public databases, including Gene Expression Omnibus (GEO; https://www.ncbi.nlm.nih.gov/gds/; accession nos. GSE74602 and GSE44076), Oncomine [Kaiser (21), Hong (22) and Sabates-Bellver (23) datasets; https://www. oncomine.org/], Human Protein Atlas (http://www.proteinatlas.org/), Gene Expressing Profiling Interactive Analysis [GEPIA; colon adenocarcinoma (COAD) and rectum adenocarcinoma (READ); http://gepia.cancer-pku.cn/].

Additionally, 18 pairs of colorectal adenocarcinoma and adjacent normal mucosal tissue samples $(5 \mathrm{~cm}$ from tumor edge) were collected from patients who had not received radiotherapy and chemotherapy prior to surgery between January 2016 and January 2017 at the Department of General Surgery, The First Affiliated Hospital of Soochow University (Suzhou, China). The clinical specimens were histologically confirmed in the pathology department. The clinicopathological features of these patients are shown in Table I. Ethics approval was granted by The Ethics Committee of The First Affiliated Hospital of Soochow University. In addition, the study was conducted in compliance with the Declaration of Helsinki. Written informed consent was obtained from all patients.

Cell lines. The human CRC cell lines (HCT116, CCL244, CaCo2, SW480, LoVo and RKO) were purchased from the Cell Bank of Type Culture Collection of Chinese Academy of Sciences. The cell cultures were routinely maintained in RPMI-1640 medium (HyClone; Cytiva) or DMEM (Hyclone; Cytiva) containing 10\% FBS (Gibco; Thermo Fisher Scientific, Inc.), $100 \mathrm{U} / \mathrm{ml}$ penicillin $\mathrm{G}$ sodium and $100 \mu \mathrm{g} / \mathrm{ml}$ streptomycin sulfate (Gibco; Thermo Fisher Scientific, Inc.) at $37^{\circ} \mathrm{C}$ in a humidified atmosphere containing $5 \% \mathrm{CO}_{2}$.

Protein extraction and western blotting. CRC tissues or cells were lysed in RIPA lysis buffer (Sigma-Aldrich; Merck KGaA). Protein concentration was quantified using a BCA Protein Assay kit (Pierce; Thermo Fisher Scientific, Inc.). Following protein separation (10 $\mu \mathrm{g} /$ lane) via $8 \%$ SDS-PAGE, proteins were transferred onto polyvinylidene difluoride membranes (Bio-Rad Laboratories, Inc.) and were then blocked in 5\% skimmed milk for $1 \mathrm{~h}$ at room temperature. Subsequently, the membranes were incubated with primary antibodies overnight at $4^{\circ} \mathrm{C}$ and then with HRP-conjugated secondary antibodies
(1:5,000; cat. nos. SA00001-2 or SA00001-1; ProteinTech Group, Inc.) for $1 \mathrm{~h}$ at room temperature. Next, the proteins were visualized using a chemiluminescence kit (EMD Millipore) and signals were quantified using ImageJ software (v1.46; National Institutes of Health). The antibodies used in this study included anti-YAP (1:1,000; cat. no. 14074; Cell Signaling Technology, Inc.), anti-E-cadherin (1:1,000; cat. no. 3195; Cell Signaling Technology, Inc.), anti-vimentin (1:1,000; cat. no. 5741; Cell Signaling Technology, Inc.), anti-Glut3 (1:1,000; cat. no. 20403-1-AP; ProteinTech Group, Inc.), anti-phosphorylated (p)-AMPK Thr172 (1:1,000; cat. no. 2535; Cell Signaling Technology, Inc.), anti-AMPK (1:1,000; cat. no. 2532; Cell Signaling Technology, Inc.) and anti-GAPDH (1:5,000; cat. no. AG019; Beyotime Institute of Biotechnology).

Plasmid and small interfering RNA (siRNA) transfection. The siRNA against YAP (specific target sequence, 5'-GGU CAGAGAUACUUCUUAAAU-3'), siRNA against Glut3 (specific target sequence, 5'-UAGCCAAAUUGGAAAGAG CTT-3') and their scrambled siRNA (target sequence, 5'-UUC UCCGAACGUGUCACGUTT-3') were synthesized by Shanghai GenePharma Co., Ltd. SW480 and HCT116 cells were transfected with the aforementioned siRNAs at a final concentration of $20 \mathrm{nM}$ using Lipofectamine ${ }^{\mathrm{TM}}$ RNAiMax (Invitrogen; Thermo Fisher Scientific, Inc.) at $4^{\circ} \mathrm{C}$ according to the manufacturer's protocol. After 48-72 h of transfection, the cells were used for subsequent experiments. Plasmids encoding the human YAP protein and the empty pcDNA3.1 vector were purchased from Shanghai GenePharma Co., Ltd. Lipofectamine ${ }^{\circledR} 2000$ (Invitrogen; Thermo Fisher Scientific, Inc.) was used to transfect HCT116 cells according to the manufacturer's instructions and as described previously (16). After 48-72 h of cell transfection, the cells were used for subsequent experiments.

Colony formation assay. In brief, $\sim 1 \times 10^{3}$ transfected SW480 or HCT116 cells were seeded in 6-well plates and cultured for 7-10 days, then fixed with 4\% paraformaldehyde (Beyotime Institute of Biotechnology) at room temperature for $30 \mathrm{~min}$ and stained with $0.1 \%$ crystal violet (Beyotime Institute of Biotechnology) at room temperature for $15 \mathrm{~min}$. The number of colonies containing $>100$ cells was determined using a light microscope (magnification, x40; Nikon Corporation).

Cell migration assay. Cell migration was assessed using Transwell $^{\mathrm{TM}}$ chambers (pore size, $8.0 \mu \mathrm{m}$; Corning, Inc.). Briefly, $200 \mu \mathrm{l}$ serum-free DMEM containing $5 \times 10^{4}$ transfected SW480 or HCT116 cells was seeded onto the filters in 24-well chambers and $750 \mu \mathrm{l}$ medium containing 10\% FBS was placed in the lower chambers as a chemoattractant. After $24 \mathrm{~h}$ at $4^{\circ} \mathrm{C}$, the filters were fixed with $4 \%$ paraformaldehyde (Beyotime Institute of Biotechnology) at room temperature for 30 min and stained with $0.1 \%$ crystal violet solution (Beyotime Institute of Biotechnology) at room temperature for $15 \mathrm{~min}$. Stained cells were visualized in three randomly selected fields using a light microscope (magnification, x200). The detailed procedure has been described previously (24).

ATP measurement. Relative ATP levels were measured using an enhanced ATP assay kit (cat. no. S0027; Beyotime Institute 
Table I. Association between YAP expression and clinicopathological parameters in patients with colorectal cancer.

\begin{tabular}{|c|c|c|c|c|}
\hline \multirow[b]{2}{*}{ Clinicopathological parameters } & \multirow[b]{2}{*}{ Total, n (\%) } & \multicolumn{2}{|c|}{ YAP expression, $\mathrm{n}(\%)$} & \multirow[b]{2}{*}{ P-value } \\
\hline & & Low, $n=9$ & High, $n=9$ & \\
\hline Age, years & & & & 0.319 \\
\hline$<65$ & $8(44.4)$ & $3(33.3)$ & $5(55.6)$ & \\
\hline$\geq 65$ & $10(55.6)$ & $6(66.7)$ & $4(44.4)$ & \\
\hline Sex & & & & 0.500 \\
\hline Male & $13(72.2)$ & $6(66.7)$ & $7(77.8)$ & \\
\hline Female & $5(27.8)$ & $3(33.3)$ & $2(22.2)$ & \\
\hline Tumor size, $\mathrm{cm}$ & & & & 0.500 \\
\hline$<5$ & $9(50.0)$ & $5(55.6)$ & $4(44.4)$ & \\
\hline$\geq 5$ & $9(50.0)$ & $4(44.4)$ & $5(55.6)$ & \\
\hline Lymph node metastasis & & & & 0.167 \\
\hline No & $11(61.1)$ & $7(77.8)$ & $4(44.4)$ & \\
\hline Yes & $7(38.9)$ & $2(22.2)$ & $5(55.6)$ & \\
\hline TNM stage & & & & 0.167 \\
\hline $\mathrm{I} / \mathrm{II}$ & $11(61.1)$ & $7(77.8)$ & $4(44.4)$ & \\
\hline III/IV & $7(38.9)$ & $2(22.2)$ & $5(55.6)$ & \\
\hline
\end{tabular}

TNM, tumor-node-metastasis; YAP, Yes-associated protein.

of Biotechnology) according to the manufacturer's instructions. Total ATP levels were calculated from the luminescence signals and were normalized by the protein concentrations.

Statistical analysis. Continuous data are presented as the mean \pm SEM, whereas categorical variables are presented as numbers and frequencies. Two-tailed paired or unpaired Student's t-tests were used for the comparison of two groups. One-way ANOVA followed by Tukey's post hoc test were used for multi-group comparisons. Fisher's exact test was used to analyze the relationships between YAP expression and clinicopathological characteristics. Spearman's correlation was used to analyze the association between the expression levels of two genes. $\mathrm{P}<0.05$ was considered to indicate a statistically significant difference.

\section{Results}

YAP is upregulated in human CRC tissue samples. In order to determine the function of YAP in CRC, its mRNA expression was analyzed using public GEO datasets. The results showed that YAP mRNA expression levels were significantly upregulated in human CRC tumor samples compared with in adjacent normal tissue samples (Fig. 1A).

The transcriptional levels of the YAP gene were also measured in public datasets from the Oncomine database. In the Kaiser (21) dataset (Fig. 1B and C), YAP mRNA expression levels were higher in cecum adenocarcinoma $(n=17)$ and colon adenocarcinoma $(n=41)$ compared with in normal colon tissue samples $(\mathrm{n}=5)\left(\mathrm{P}=8.18 \times 10^{-9}\right.$ and $\mathrm{P}=2.89 \times 10^{-12}$, respectively). Moreover, in the Hong (22) Oncomine dataset (Fig. 1D), the mRNA expression levels of YAP in colorectal carcinoma tissue $(n=70)$ were higher than in normal colon $\left(n=12 ; P=9.10 \times 10^{-14}\right)$.
In addition, YAP mRNA expression levels in rectal adenoma tissue $(n=7)$ were higher than those in normal colorectal tissue in the Sabates-Bellver (23) Oncomine dataset (Fig. 1E; $\left.\mathrm{P}=2.10 \times 10^{-8}\right)$. However, no significant differences were observed in the expression of the YAP gene between normal ascending colon, descending colon, sigmoid colon, transverse colon and normal rectum samples (Fig. 1E).

Consistent with the public datasets, YAP protein expression levels in clinical samples were significantly higher in CRC tissue than in normal tissue (Fig. 1F and G; $n=18$ in each group). The relationship between YAP expression levels and the clinicopathological parameters of patients with CRC was then evaluated. Notably, there were no significant associations between YAP and age, sex, tumor size, lymph node metastasis or TNM stage (Table I). In order to further determine the clinical relevance of YAP, the expression of the YAP protein in histological specimens from the Human Protein Atlas program was analyzed. The results indicated a weak expression of YAP in normal colorectal tissue but a strong expression in CRC tissue (Fig. 1H). Collectively, these results suggested that YAP expression was upregulated in human CRC tissue both at the mRNA and the protein levels, regardless of the tumor location.

YAP expression in the CRC cell lines. The expression of the YAP protein was examined in six CRC cell lines (HCT116, CCL244, CaCo2, SW480, LoVo and RKO) using western blot analysis. The results indicated that the YAP protein was expressed at high levels in the SW480 and $\mathrm{CaCo} 2$ cell lines but at relatively lower levels in HCT116 and RKO cells (Fig. 2A). Therefore, the expression of YAP was silenced in SW480 cells (since they displayed high endogenous expression of YAP) and overexpressed in HCT116 cells (due to their low endogenous expression of YAP protein). The efficiency of YAP gene 
A

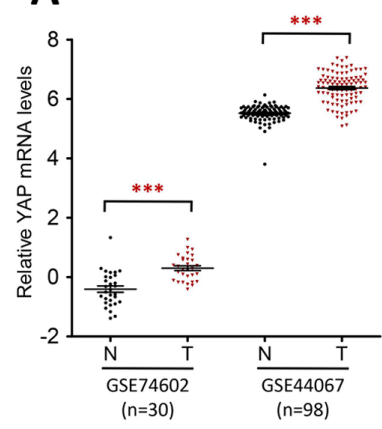

D

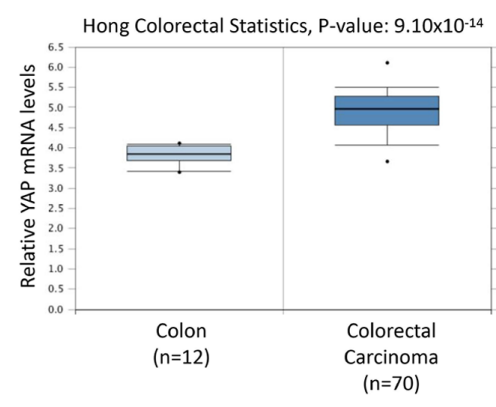

$\mathbf{F}$

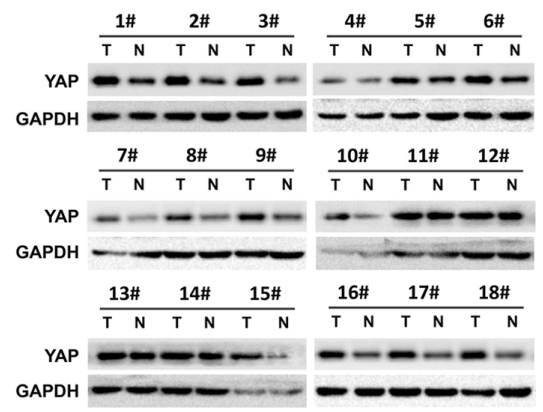

B

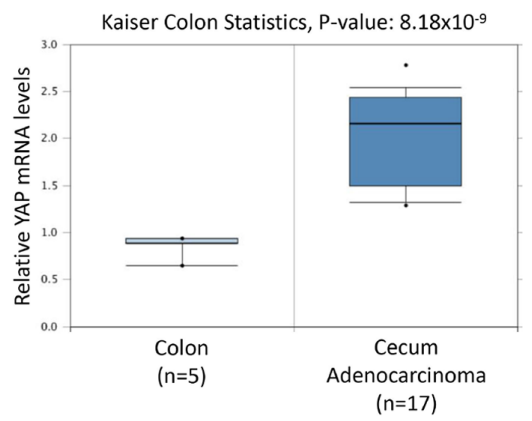

C

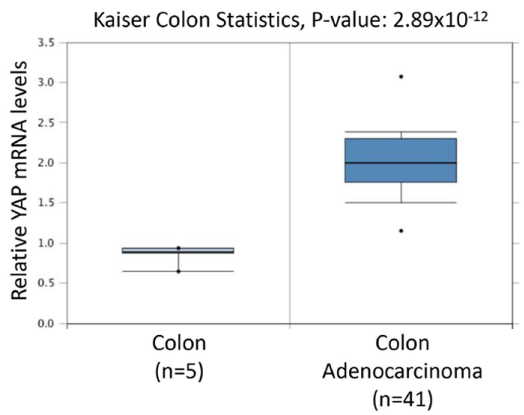

E

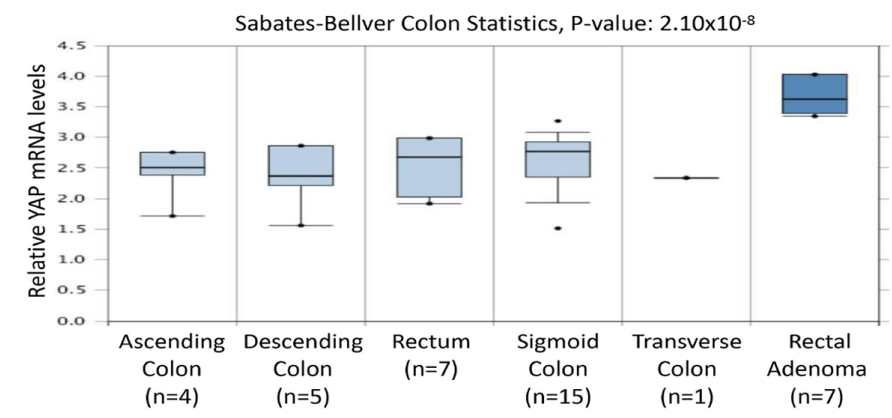

G

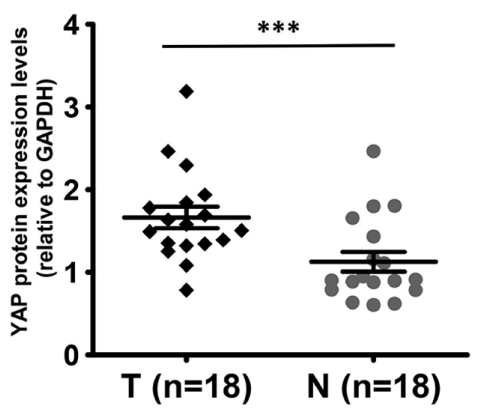

H

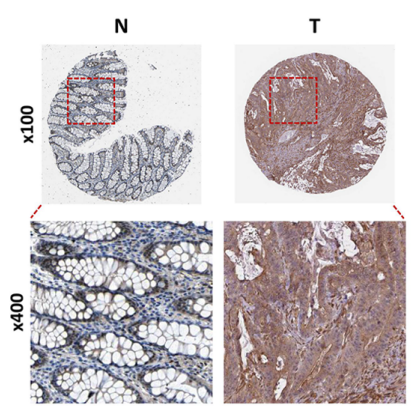

Figure 1. YAP is upregulated in human CRC. (A) Column dot plots indicating a difference in YAP mRNA expression between human CRC tissue and normal tissue based on two public microarray datasets (accession nos. GSE74602 and GSE44067). (B and C) Relative YAP mRNA expression in (B) normal colon and cecum adenocarcinoma tissue or (C) normal colon and colon adenocarcinoma tissue in the Oncomine dataset by Kaiser et al (21). (D) Relative YAP mRNA expression in normal colon or colorectal carcinoma tissue in the Oncomine dataset by Hong et al (22). (E) Relative YAP mRNA expression in normal ascending colon, descending colon, rectum, sigmoid colon, transverse colon or rectal adenoma tissue in the Oncomine dataset by Sabates-Bellver et al (23). (F) Western blot analysis of YAP protein expression levels in 18 pairs of CRC tumor and normal adjacent tissue samples. (G) Semi-quantitative analysis of YAP expression levels (relative to GAPDH) in 18 pairs of CRC tumor and normal adjacent tissue samples. ${ }^{* * *} \mathrm{P}<0.001$, two-tailed paired Student's t-test. (H) YAP expression in human colorectal tumor and normal tissue. Images were taken from the Human Protein Atlas online database (magnification, $\mathrm{x} 100$ and $\mathrm{x} 400$ ) and are available from https://www.proteinatlas.org/ENSG00000137693-YAP1/tissue/colon\#img and https://www.proteinatlas. org/ENSG00000137693-YAP1/pathology/colorectal+cancer\#img. CRC, colorectal cancer; GEPIA, Gene Expression Profiling Interactive Analysis; N, normal adjacent tissue; T, tumor tissue; YAP, Yes-associated protein.

knockdown and overexpression was confirmed using western blot analysis. As shown in Fig. 2B, YAP protein expression was significantly decreased in SW480 cells transfected with YAP-specific siRNA compared with that in cells transfected with siRNA NC. Conversely, YAP protein expression was significantly increased in HCT116 cells transfected with a plasmid encoding the human YAP protein compared with a control vector (Fig. 2C).

YAP enhances the proliferation and migration of CRC cells in vitro. In order to determine whether YAP played an oncogenic role in $\mathrm{CRC}$, functional experiments were performed to evaluate the effects of YAP on the proliferation and migration of CRC cells in vitro. Colony formation assays demonstrated that YAP silencing inhibited the ability of SW480 cells to form colonies (Fig. 3A). By contrast, the proliferation of HCT116 cells was significantly enhanced following the overexpression of YAP (Fig. 3B).

Transwell assays were conducted to measure the migratory ability of CRC cells. The results suggested that the migration of SW480 cells was significantly impaired following transfection with YAP-specific siRNA compared with NC siRNA (Fig. 3C). However, the migration of HCT116 cells transfected with the YAP overexpression plasmid doubled compared with cells transfected with the empty vector (Fig. 3D). These results indicated that YAP served an oncogenic role in CRC cells. 

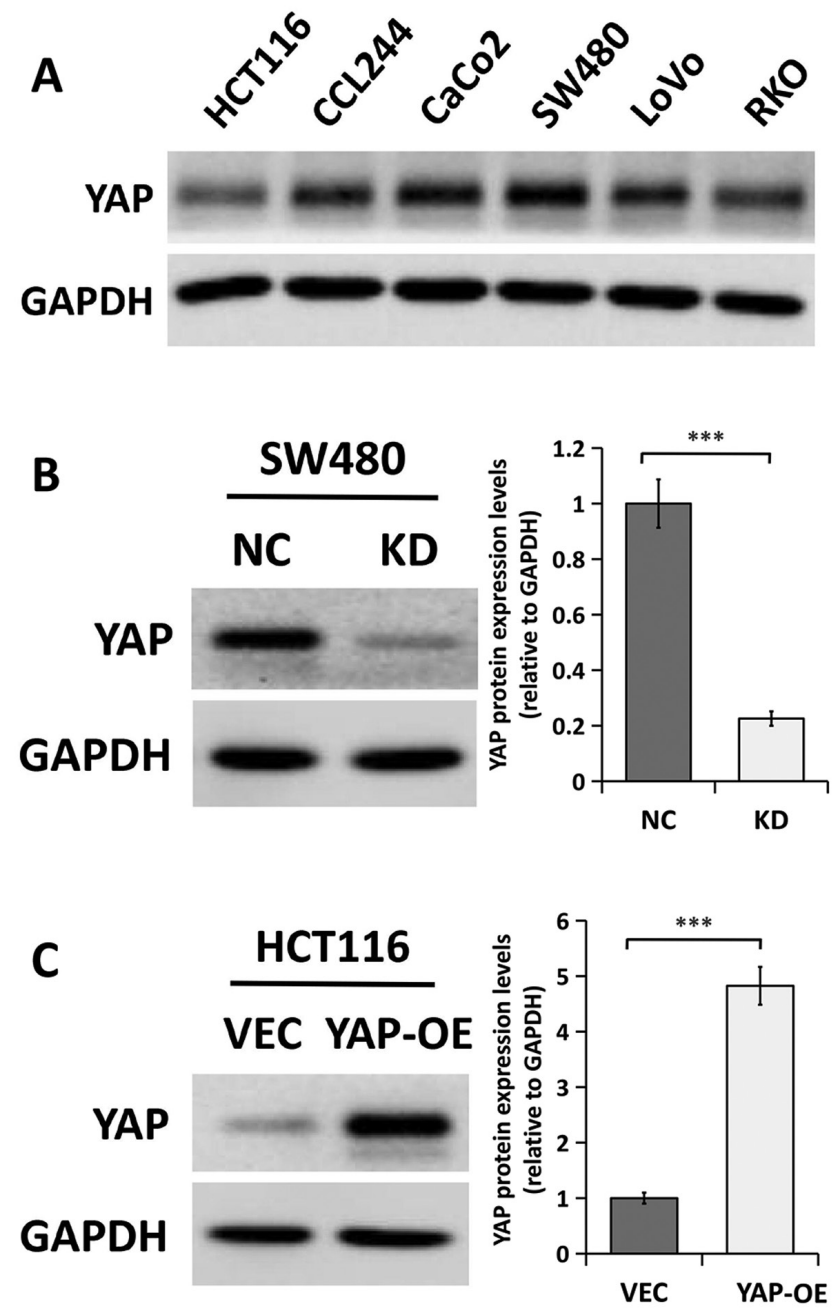

Figure 2. YAP protein expression levels in human CRC cells. (A) Western blot analysis of YAP protein levels in six CRC cell lines (HCT116, CCL244, CaCo2, SW480, LoVo and RKO) performed in triplicate. (B) Western blot analysis of YAP protein expression in SW480 cells transfected with NC or $\mathrm{KD}$. The bands were semi-quantified and the results are presented as the mean \pm SEM. $n=3$. (C) Western blot analysis of YAP in HCT116 cells transfected with VEC or YAP-OE. The bands were semi-quantified and the results are presented as the mean \pm SEM. $n=3 .{ }^{* * *} \mathrm{P}<0.001$, two-tailed unpaired Student's t-test. CRC, colorectal cancer; KD, YAP siRNA knockdown; NC, siRNA negative control; OE, overexpression; VEC, empty vector; YAP, Yes-associated protein; siRNA, small interfering RNA.

Expression of YAP is associated with the EMT. Considering that YAP promotes the migration of CRC cells and that EMT is an early event in the metastasis of cancer $(25,26)$, the regulation of EMT progression by YAP was examined. The correlation between YAP mRNA expression levels and EMT-related gene expression was analyzed in CRC specimens from GEPIA datasets. The results showed that YAP mRNA expression levels were positively correlated with those of EMT-related genes, including vimentin (Fig. 4A; $\rho=0.37$; $\mathrm{P}=4.2 \times 10^{-13}$ ), cadherin-2 (also known as N-cadherin; Fig. 4B; $\rho=0.35 ; P=8.4 \times 10^{-12}$ ), Snail family transcriptional repressor 1 (SNAII; Fig. 4C; $\rho=0.36, \mathrm{P}=7 \times 10^{-13}$ ), SNAI2 (also known as Slug; Fig. 4D; $\left.\rho=0.4 ; \mathrm{P}=1.3 \times 10^{-15}\right)$, zinc finger E-box binding homeobox (ZEB1; Fig. 4E; $\rho=0.51 ; \mathrm{P}=4.2 \times 10^{-26}$ ) and $Z E B 2$ (Fig. 4F; $\rho=0.43 ; \mathrm{P}=2.5 \times 10^{-18}$ ). Furthermore, the expression levels of the epithelial marker E-cadherin and mesenchymal marker vimentin were detected using western blot analysis. As shown in Fig. 4G, YAP silencing significantly increased the expression of E-cadherin and decreased that of vimentin. By contrast, YAP overexpression had the opposite effect on the expression of these EMT markers (Fig. 4H). These results indicated that YAP played a role in the regulation of EMT in CRC cells.

$Y A P$ regulates the Glut $3 / A M P K$ pathway in CRC cells. It has previously been reported that YAP transcriptionally regulates the $S L C 2 A 3$ gene (encoding the Glut3 protein) and that the expression levels of these two molecules are positively correlated in CRC tissue $(27,28)$. To confirm this, the correlation between the mRNA expression levels of YAP and SLC2A3 in CRC specimens was examined using GEPIA datasets. A positive correlation was observed between the mRNA expression of $Y A P$ and that of $S L C 2 A 3$ (Fig. $5 \mathrm{~A} ; \rho=0.3 ; \mathrm{P}=7.4 \times 10^{-9}$ ). Furthermore, the results from western blot analysis indicated that YAP silencing significantly decreased the expression of Glut3 (Fig. 5B). By contrast, YAP overexpression significantly increased Glut3 expression levels (Fig. 5C). Moreover, the regulation of Glut 3 by YAP was also evidenced by the fact that YAP silencing increased the levels of p-AMPK and decreased cellular ATP levels (Fig. 5B and D). Notably, YAP overexpression had the opposite effects on the levels of p-AMPK and ATP (Fig. 5C and D).

In order to further explore whether Glut3 was involved in the regulation of YAP during cell migration, Glut3-specific siRNA (siGlut3) was used to silence the expression of Glut3 in YAP-overexpressing HCT116 cells. The results of western blot analysis showed that Glut3 protein expression levels were markedly decreased in cells transfected with siRNA compared with the control group (Fig. 5E). As shown in Fig. 5F, YAP overexpression increased Glut3 expression compared with the control group, which was then decreased in HCT116 cells following Glut3 siRNA treatment. Moreover, migration assays suggested that the overexpression of YAP significantly enhanced the migration ability of cells transfected with a control siRNA, but this effect was inhibited following co-transfection with siGlut3 (Fig. 5G).

\section{Discussion}

Several lines of evidence have suggested that the Hippo signaling pathway has a tumor-suppressive role and that impaired Hippo signaling contributes to the development and progression of cancer, including CRC (5-8). YAP, as a major downstream effector in the Hippo signaling pathway, has been identified as an oncogene that is frequently overexpressed in several common human cancer types (9-16). The upregulation of YAP has been reported to promote the proliferation, migration and chemoresistance of tumor cells in multiple types of cancer, including pancreatic cancer (9), breast cancer (10), hepatocellular carcinoma (11), esophageal cancer (12), cervical cancer (13), ovarian cancer (14) and gastric cancer $(15,16)$. Although previous studies focusing on the role of YAP in CRC have shown that YAP is expressed at high levels in CRC tissue, and promotes the proliferation and migration of CRC cells (17-20), the underlying mechanism is not fully understood and remains to be examined. Using public databases 
A

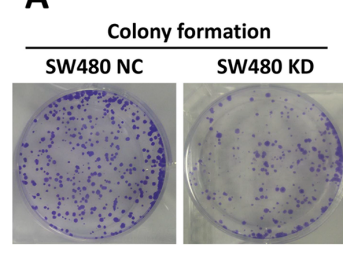

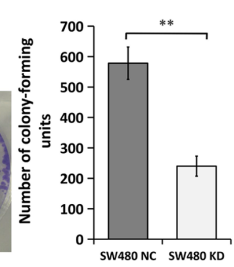

B
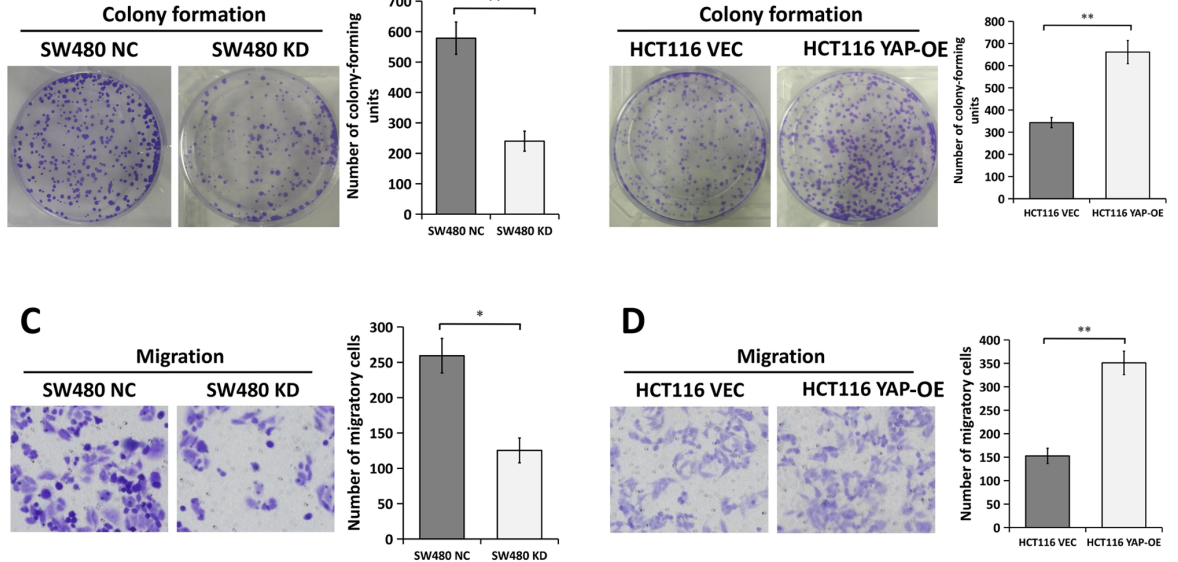

Figure 3. Effects of YAP on CRC cell proliferation and migration in vitro. Colony formation assays were performed in (A) SW480 cells transfected with NC or KD and (B) HCT116 cells transfected with VEC or YAP-OE. Representative images of the wells are presented (left). The number of colony-forming units (right) is presented as the mean \pm SEM. $n=3$. Migration assays were conducted in (C) SW480 cells transfected with NC or KD and (D) HCT116 cells transfected with VEC or YAP-OE. Representative images are presented (left). Magnification, x200. The number of migratory cells (right) is presented as the mean \pm SEM. $n=3 .{ }^{~} \mathrm{P}<0.05,{ }^{* *} \mathrm{P}<0.01$, two-tailed unpaired Student's t-test. CRC, colorectal cancer; KD, YAP siRNA knockdown; NC, siRNA negative control; OE, overexpression; VEC, empty vector; YAP, Yes-associated protein; siRNA, small interfering RNA.

A

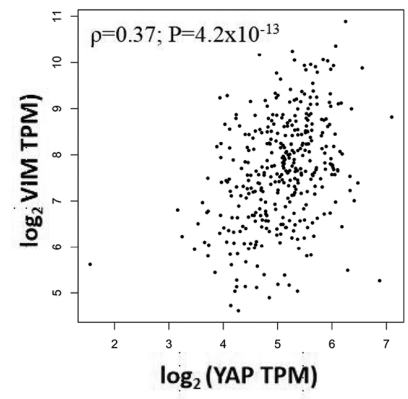

D

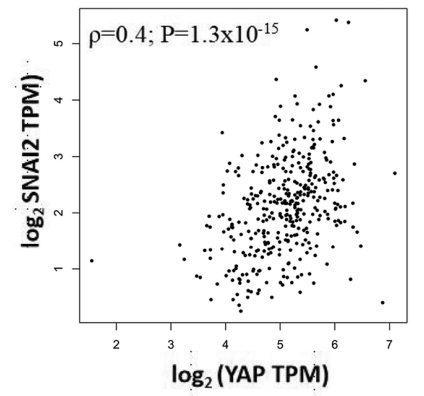

B

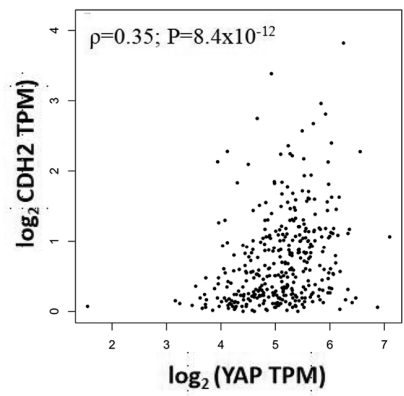

E

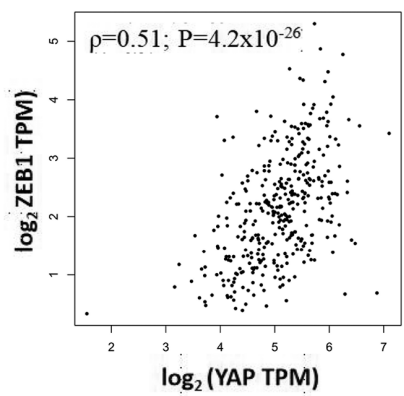

C

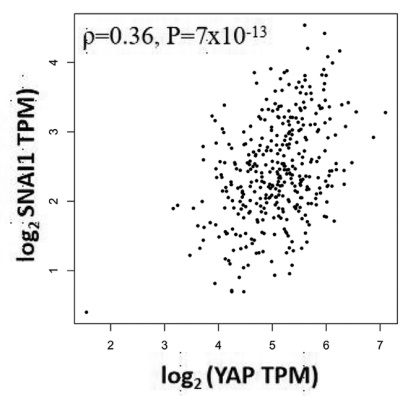

$\mathbf{F}$

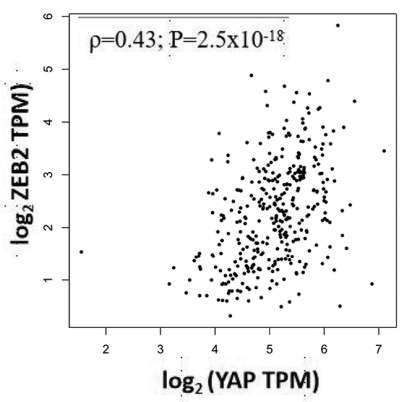

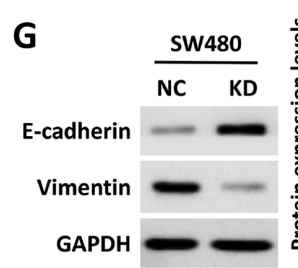
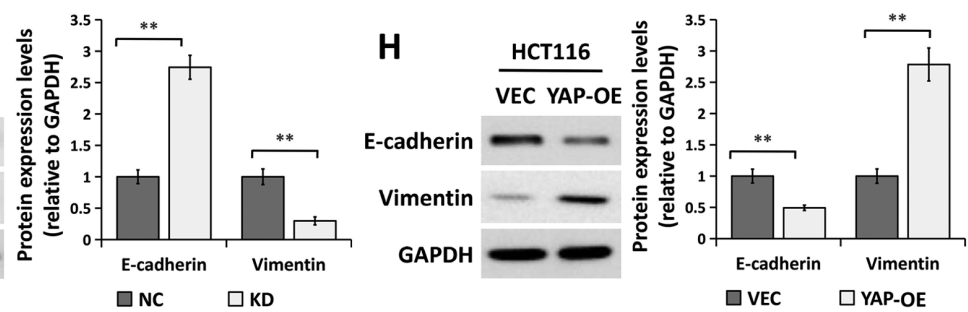

Figure 4. YAP is associated with EMT. (A) Correlation analysis of YAP1 and VIM gene expression levels in patients with CRC using GEPIA datasets (COAD and READ). (B) Correlation analysis of $Y A P 1$ and $C D H 2$ gene expression levels using CRC datasets from GEPIA. (C) Correlation analysis between $Y A P 1$ and SNAIl gene expression in patients with CRC using GEPIA datasets. (D) Correlation analysis between YAPI and SNAI2 gene expression in patients with CRC using GEPIA datasets. (E) Correlation analysis between YAPI and ZEB1 gene expression in patients with CRC using GEPIA datasets. (F) Correlation analysis between $Y A P 1$ and ZEB2 gene expression in patients with CRC using GEPIA datasets. Western blot analysis of E-cadherin and vimentin protein expression in (G) SW480 cells transfected with NC or KD and (H) HCT116 transfected with VEC or YAP-OE. GAPDH was used as a loading control. The bands were semi-quantified and the results are presented as the mean \pm SEM (right). $n=3$. ${ }^{* *} \mathrm{P}<0.01$, two-tailed unpaired Student's t-test. CRC, colorectal cancer, $C D H 2$, N-cadherin; GEPIA, Gene Expression Profiling Interactive Analysis; SNAI1/2, Snail family transcriptional repressor 1/2; ZEB1/2, zinc finger E-box binding homeobox 1/2; TPM, transcripts per million; KD, YAP siRNA knockdown; NC, siRNA negative control; OE, overexpression; VEC, empty vector; YAP, Yes-associated protein; siRNA, small interfering RNA; COAD, colon adenocarcinoma; READ, rectum adenocarcinoma. 

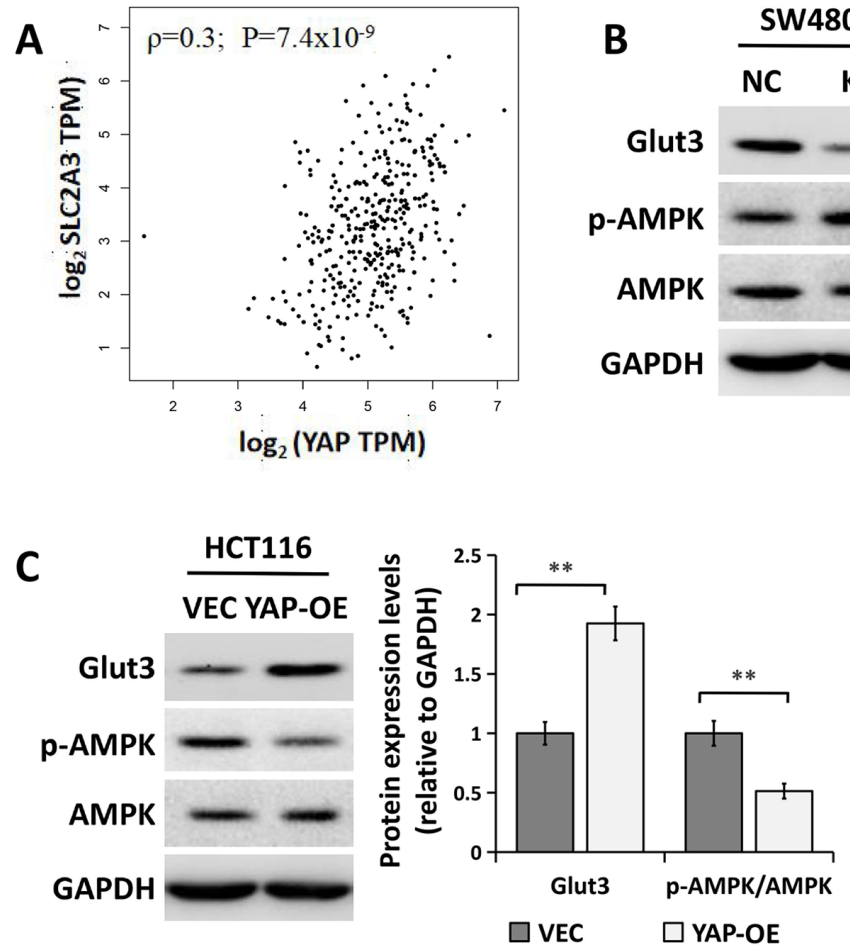

D
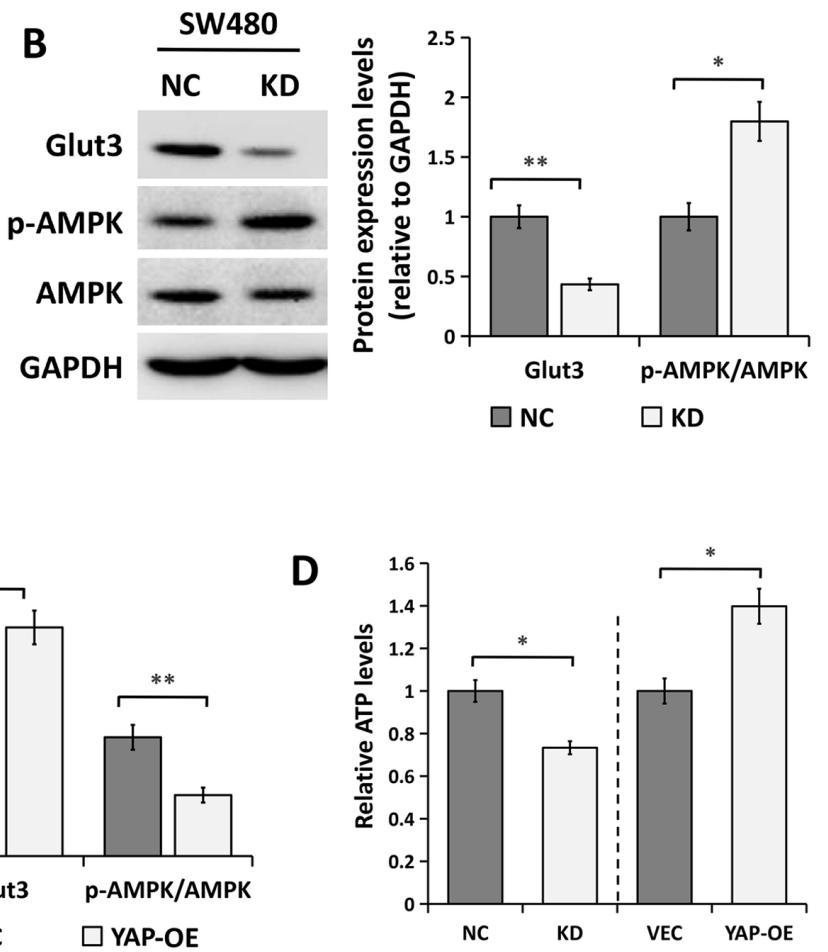

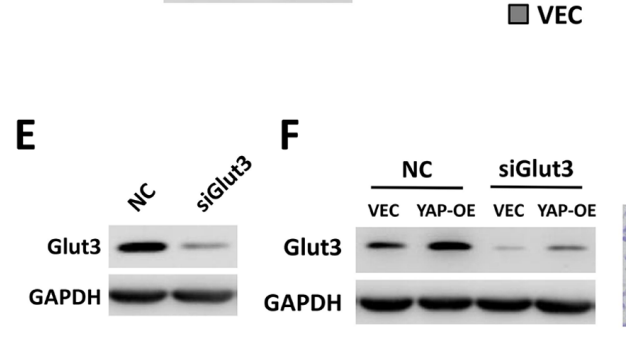

F
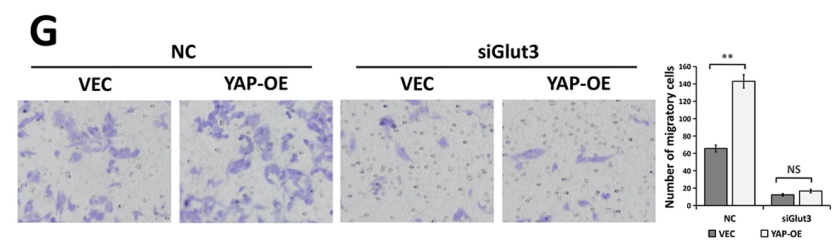

Figure 5. YAP regulates the Glut3/AMPK pathway in CRC cells. (A) Correlation analysis between YAP1 and SLC2A3 (Glut3) gene expression levels in patients with CRC using GEPIA datasets. Western blot analysis of Glut3, p-AMPK and AMPK in (B) SW480 cells transfected NC or KD and (C) HCT116 cells transfected with VEC or YAP-OE. GAPDH was used as a loading control. The bands were semi-quantified and the results are presented as the mean \pm SEM (right). $\mathrm{n}=3$. (D) Relative ATP levels in SW480 cells transfected with NC or KD and HCT116 cells transfected with VEC or YAP-OE. (E) Western blot analysis of Glut3 protein expression in HCT116 cells transfected with siGlut3 or NC. (F) Western blot analysis of Glut3 expression in HCT116 cells co-transfected with siGlut3 or NC together with YAP-OE or VEC. (G) Migration ability of HCT116 cells in HCT116 cells co-transfected with siGlut3 or NC together with YAP-OE or VEC. Representative images are presented (left). Magnification, $\mathrm{x} 200$. The number of migratory cells (right) is presented as the mean $\pm \mathrm{SEM}$. $\mathrm{n}=3$. " $\mathrm{P}<0.05$; ${ }^{* *} \mathrm{P}<0.01$; NS, not significant, two-tailed unpaired Student's t-test. AMPK, AMP-activated protein kinase; CRC, colorectal cancer; Glut3, glucose transporter 3; KD, YAP siRNA knockdown; NC, siRNA negative control; p-; phosphorylated; OE, overexpression; SLC2A3, solute carrier family 2 member 3; VEC, empty vector; YAP, Yes-associated protein; siRNA, small interfering RNA.

and western blot analysis of clinical samples, the present study demonstrated that both the mRNA and protein expression levels of YAP were significantly upregulated in human CRC tissue compared with in normal tissue. Moreover, in vitro data showed that YAP not only regulated the proliferative ability of CRC cells but also enhanced their migration, indicating that the upregulation of YAP contributed to the proliferation and migration of CRC cells after malignant transformation.

The induction of EMT has been proposed to play a critical role in tumor metastasis and stemness of cancer cells, and is involved in tumor initiation $(20,25,26)$. EMT is triggered by several transcription factors, including zinc-finger binding transcription factors, such as Snaill and -2 (also known as Slug), and ZEB1 and $-2(25,29)$. The progression of EMT is accompanied by the upregulation of mesenchymal markers, including vimentin and $\mathrm{N}$-cadherin, and the downregulation of epithelial markers, such as E-cadherin $(25,29)$. In the present study, the expression of the YAP gene was positively correlated with the EMT markers VIM and $C D H 2$, as well as the EMT-inducing transcription factors SNAII, SNAI2, ZEB1 and ZEB2 in CRC specimens from public datasets. Furthermore, it was demonstrated that YAP silencing caused an increase in the protein expression of the epithelial marker E-cadherin and a decrease in that of the mesenchymal marker vimentin in CRC cells. However, the overexpression of YAP resulted in the opposite effect. These findings indicated that YAP might serve a crucial role in the regulation of EMT.

Previous studies have demonstrated that YAP expression may be positively correlated with Glut 3 and could regulate its transcription in CRC tissue $(27,28)$. The present study confirmed the positive correlation between the expression levels of the $Y A P$ and $S L C 2 A 3$ genes in CRC specimens from public datasets. Furthermore, it was demonstrated that YAP promoted the expression of the Glut3 protein, leading to an increase in ATP levels, which may lead to decreased AMPK phosphorylation. Furthermore, a recent study indicated that 
Glut3 had a greater impact on the proliferation of CRC cells than Glut1 under glucose-limiting stress conditions, and that high Glut3 expression markedly increased the sensitivity of CRC cells to treatment with vitamin C (30), highlighting the therapeutic potential of targeting Glut 3 in the treatment of CRC.

There are certain limitations in the present study. Firstly, the expression of YAP protein was measured using western blot analysis in a small number of CRC tissue samples. More samples should be examined and the correlation between the expression of YAP and clinicopathological parameters of patients with CRC should be assessed. Secondly, the expression of Glut3 or AMPK activity was not detected in CRC tissue. Thirdly, the effect of YAP on CRC tumorigenesis and metastasis was not examined in vivo in the present study, and studies involving subcutaneous xenograft models should be further performed in the future.

In conclusion, the present study demonstrated the upregulation of YAP in CRC tissue, and emphasized the regulatory role of YAP in the proliferation and migration of CRC cells. Moreover, a potential mechanism was revealed through which YAP could induce proliferation and migration of CRC cells and EMT progression via Glut3/AMPK signaling. These findings may provide insight into novel approaches for the treatment of CRC involving YAP targeting.

\section{Acknowledgements}

Not applicable.

\section{Funding}

The present study was supported by the Project of National Science Foundation of Jiangsu Province of China (grant no. BK20161225) and the Project of Science and Technology Plan of Suzhou of China (grant no. SYS2019050).

\section{Availability of data and materials}

The datasets used and/or analyzed during the present study are available from the corresponding author on reasonable request. Additionally, the datasets generated and/or analyzed during the current study are available from public databases, including Gene Expression Omnibus (https://www.ncbi.nlm. nih.gov/gds/; accession nos. GSE74602 and GSE44076), Oncomine [Kaiser (21), Hong (22) and Sabates-Bellver (23) datasets; thttps://www.oncomine.org/], Human Protein Atlas (http://www.proteinatlas.org/), Gene Expressing Profiling Interactive Analysis [colon adenocarcinoma (COAD) and rectum adenocarcinoma (READ) datasets; http://gepia. cancer-pku.cn/].

\section{Authors' contributions}

LJ, JZ and QX conducted the research, analyzed the data and wrote the manuscript. BW, YY, LS, XW, DZ, LG and SS contributed to data collection and analysis. XZ designed the study and supervised the preparation of the manuscript. LJ and JZ confirm the authenticity of the data. All authors have read and approved the final manuscript.

\section{Ethics approval and consent to participate}

This study was approved by The Biomedical Research Ethics Committee of The First Affiliated Hospital of Soochow University. The experiments performed using human tissue were in compliance with the Declaration of Helsinki. Written informed consent was obtained from all patients in this study.

\section{Patient consent for publication}

Not applicable.

\section{Competing interests}

The authors declare that they have no competing interests.

\section{References}

1. Siegel RL, Miller KD and Jemal A: Cancer statistics, 2019. CA Cancer J Clin 69: 7-34, 2019.

2. Arnold M, Sierra MS, Laversanne M, Soerjomataram I, Jemal A and Bray F: Global patterns and trends in colorectal cancer incidence and mortality. Gut 66: 683-691, 2017.

3. Lintoiu-Ursut B, Tulin A and Constantinoiu S: Recurrence after hepatic resection in colorectal cancer liver metastasis-review article. J Med Life 8: 12-14, 2015.

4. Liu S, Zheng R, Zhang M, Zhang S, Sun X and Chen W: Incidence and mortality of colorectal cancer in China, 2011. Chin J Cancer Res 27: 22-28, 2015.

5. Moroishi T, Hansen CG and Guan KL: The emerging roles of YAP and TAZ in cancer. Nat Rev Cancer 15: 73-79, 2015.

6. Zhao B, Tumaneng K and Guan KL: The Hippo pathway in organ size control, tissue regeneration and stem cell self-renewal. Nat Cell Biol 13: 877-883, 2011.

7. Harvey KF, Zhang X and Thomas DM: The Hippo pathway and human cancer. Nat Rev Cancer 13: 246-257, 2013.

8. Wierzbicki PM and Rybarczyk A: The Hippo pathway in colorectal cancer. Folia Histochem Cytobiol 53: 105-119, 2015.

9. Zhou Q, Bauden M, Andersson R, Hu D, Marko-Varga G, Xu J, Sasor A, Dai H, Pawłowski K, Said Hilmersson K, et al: YAP1 is an independent prognostic marker in pancreatic cancer and associated with extracellular matrix remodeling. J Transl Med 18: 77, 2020.

10. Zhang Z, Qiu N, Yin J, Zhang J, Liu H, Guo W, Liu M, Liu T, Chen D, Luo K, et al: SRGN crosstalks with YAP to maintain chemoresistance and stemness in breast cancer cells by modulating HDAC2 expression. Theranostics 10: 4290-4307, 2020.

11. Zhou Y, Wang Y, Zhou W, Chen T, Wu Q, Chutturghoon VK, Lin B, Geng L, Yang Z, Zhou L and Zheng S: YAP promotes multi-drug resistance and inhibits autophagy-related cell death in hepatocellular carcinoma via the RAC1-ROS-mTOR pathway. Cancer Cell Int 19: 179, 2019.

12. Qu Y, Zhang L, Wang J, Chen P, Jia Y, Wang C, Yang W, Wen Z, Song Q, Tan B and Cheng Y: Yes-associated protein (YAP) predicts poor prognosis and regulates progression of esophageal squamous cell cancer through epithelial-mesenchymal transition. Exp Ther Med 18: 2993-3001, 2019.

13. He C, Mao D, Hua G, Lv X, Chen X, Angeletti PC, Dong J, Remmenga SW, Rodabaugh KJ, Zhou J, et al: The Hippo/YAP pathway interacts with EGFR signaling and HPV oncoproteins to regulate cervical cancer progression. EMBO Mol Med 7: 1426-1449, 2015.

14. Wei X, Jia Y, Lou H, Ma J, Huang Q, Meng Y, Sun C, Yang Z, Li X, Xu S, et al: Targeting YAP suppresses ovarian cancer progression through regulation of the PI3K/Akt/mTOR pathway. Oncol Rep 42: 2768-2776, 2019.

15. Kang W, Tong JH, Chan AW, Lee TL, Lung RW, Leung PP, So KK, Wu K, Fan D, Yu J, et al: Yes-associated protein 1 exhibits oncogenic property in gastric cancer and its nuclear accumulation associates with poor prognosis. Clin Cancer Res 17: 2130-2139, 2011.

16. Lu T, Sun L and Zhu X: Yes-associated protein enhances proliferation and attenuates sensitivity to cisplatin in human gastric cancer cells. Biomed Pharmacother 105: 1269-1275, 2018. 
17. Avruch J, Zhou D and Bardeesy N: YAP oncogene overexpression supercharges colon cancer proliferation. Cell Cycle 11: 1090-1096, 2012.

18. Sun Z, Ou C, Liu J, Chen C, Zhou Q, Yang S, Li G, Wang G, Song J, Li Z, et al: YAP1-induced MALAT1 promotes epithelial-mesenchymal transition and angiogenesis by sponging miR-126-5p in colorectal cancer. Oncogene 38: 2627-2644, 2019.

19. Chen C, Zhu D, Zhang H, Han C, Xue G, Zhu T, Luo J and Kong L: YAP-dependent ubiquitination and degradation of $\beta$-catenin mediates inhibition of Wnt signalling induced by Physalin F in colorectal cancer. Cell Death Dis 9: 591, 2018.

20. Ling HH, Kuo CC, Lin BX, Huang YH and Lin CW: Elevation of YAP promotes the epithelial-mesenchymal transition and tumor aggressiveness in colorectal cancer. Exp Cell Res 350: 218-225, 2017.

21. Kaiser S, Park YK, Franklin JL, Halberg RB, Yu M, Jessen WJ, Freudenberg J, Chen X, Haigis K, Jegga AG, et al: Transcriptional recapitulation and subversion of embryonic colon development by mouse colon tumor models and human colon cancer. Genome Biol 8: R131, 2007.

22. Hong Y, Downey T, Eu KW, Koh PK and Cheah PY: A 'metastasis-prone' signature for early-stage mismatch-repair proficient sporadic colorectal cancer patients and its implications for possible therapeutics. Clin Exp Metastasis 27: 83-90, 2010.

23. Sabates-Bellver J, Van der Flier LG, de Palo M, Cattaneo E, Maake C, Rehrauer H, Laczko E, Kurowski MA, Bujnicki JM, Menigatti M, et al: Transcriptome profile of human colorectal adenomas. Mol Cancer Res 5: 1263-1275, 2007.

24. Sun L, Lu T, Tian K, Zhou D, Yuan J, Wang X, Zhu Z, Wan D, Yao Y, Zhu X and He S: Alpha-enolase promotes gastric cancer cell proliferation and metastasis via regulating AKT signaling pathway. Eur J Pharmacol 845: 8-15, 2019.
25. Gonzalez DM and Medici D: Signaling mechanisms of the epithelial-mesenchymal transition. Sci Signal 7: re8, 2014.

26. Tania M, Khan MA and Fu J: Epithelial to mesenchymal transition inducing transcription factors and metastatic cancer. Tumour Biol 35: 7335-7342, 2014.

27. Wang W, Xiao ZD, Li X, Aziz KE, Gan B, Johnson RL and Chen J: AMPK modulates Hippo pathway activity to regulate energy homeostasis. Nat Cell Biol 17: 490-499, 2015.

28. Kuo CC, Ling $\mathrm{HH}$, Chiang MC, Chung $\mathrm{CH}$, Lee WY, Chu CY, Wu YC, Chen CH, Lai YW, Tsai IL, et al: Metastatic colorectal cancer rewrites metabolic program through a Glut3-YAP-dependent signaling circuit. Theranostics 9: 2526-2540, 2019.

29. Peinado H, Portillo F and Cano A: Transcriptional regulation of cadherins during development and carcinogenesis. Int J Dev Biol 48: 365-375, 2004.

30. Dai W, Xu Y, Mo S, Li Q, Yu J, Wang R, Ma Y, Ni Y, Xiang W, Han L, et al: GLUT3 induced by AMPK/CREB1 axis is key for withstanding energy stress and augments the efficacy of current colorectal cancer therapies. Signal Transduct Target Ther 5: 177, 2020.

This work is licensed under a Creative Commons Attribution-NonCommercial-NoDerivatives 4.0 International (CC BY-NC-ND 4.0) License. 